\section{Warum ADHS keine Krankheit ist - eine Streitschrift}

Amrei Wittwer. Warum ADHS keine Krankheit ist - eine Streitschrift. Stuttgart: S. Hirzel Verlag 2019. 310 Seiten, kartoniert, 29,00 Euro, ISBN 978-377-762-761-8

Das Buch „Warum ADHS keine Krankheit ist - eine Streitschrift" von Amrei Wittwer ist ein wichtiges Werk, dass sehr kritisch mit der Diagnose ADHS, mit dem Umgang der Medizin mit Kindern mit Aufmerksamkeits- und Aktivitätsthemen umgeht. In der Tat teilt auch der Rezensent die Skepsis der Autorin, wenn zum Beispiel aus den USA berichtet wird, dass mindestens $20 \%$ aller Kinder und Jugendlichen an einem sogenannten ADHS litten und in manchen Regionen in Europa, wie es auch die Autorin richtig wiedergibt, angeblich jedes 4 . Kind eine ADHS-Diagnose aufweise. In der Tat ist die Diskussion, ob Versagen der Pädagogik, mangelnde Unterstützung durch Familien und fehlende Resilienzfaktoren manche Kinder in der heutigen Umwelt gegenüber hilflos macht. Ob Kinder psychische Themen entwickeln hängt in der Tat auch nach neuesten Studien aus Deutschland vom Elternhaus, nämlich psychischen Erkrankungen der Eltern und zum Beispiel niedrigem Einkommen der Familien (!) ab. Auf der anderen Seite muss auch die Autorin durchaus zur Kenntnis nehmen, dass es Hinweise für genetische Prädeterminanten von Verhalten gibt, das Verhalten aus einem Zusammenspiel von Genetik, Biologie, Umwelt und Erziehung zustande kommt. Verhalten als Spiegel unserer Gesellschaft, kindliche Entwicklung als Maßstab und Gradmesser unserer Umweltentwicklungen und des Umgangs untereinander! Es ist nicht zufällig, dass gerade auch Gesellschaften mit einem hohen Grad an Ungleichheit, d. h. Gesellschaften in denen sehr arme Menschen und sehr reiche Menschen im selben Land leben, eine geringere Lebenserwartung und eine höhere Prävalenz von psychischen Erkrankungen und psychischen Themen aufweisen als etwa Länder in denen bezüglich des Einkommens und des Bildungsstatus eher ein homogeneres Bild vorhanden ist.

Der kritische Umgang der Autorin zum Beispiel auch bezüglich der Therapie mit stimulierenden Drogen (Medikamenten!) und der Frage ob Medikamente überhaupt wirken und den Ursachen mit Diagnosekriterien, mit denen ADHS diagnostiziert wird, wirkt manchmal im Buch pharisäerhaft, überzogen und ist nicht immer evidenzbasiert. Auf der anderen Seite ist der Hinweis in Teil 4, dass einem sorgfältigen Abwegen, wann denn nun Stimulanzien verschrieben werden sollten und wann nicht, ein wichtiger Hinweis und ein wichtiger Ruf nach einem gesunden Umgang mit dem Thema ADHS. Teil 5 des Buches widmet sich entsprechend auch psychologischen und pädagogischen Förderbausteinen, der nicht medikamentösen Therapie und dem generellen Thema was wir alle, d. h. unsere Gesellschaft tun können, um Kindern mit Themen der Aufmerksamkeitsdefizit und Hyperaktivität besser zu helfen. Ein Para- digmenwechsel weg von der Medizin zur Pädagogik wird gefordert und ist auch in den Augen des Rezensenten begrüßenswert und überfällig! Andererseits betont nach der persönlichen Einschätzung des Rezensenten die Autorin leider die psychosozialen, soziodemographischen und gesellschaftlichen Hintergründe noch zu wenig: Viele Partner in den Gesundheitswesen vieler Länder profitieren von der Diagnose ADHS: Sowohl Diagnostiker als auch Therapeuten, die Pharmaindustrie, pädagogische Einrichtungen aber vielleicht auch die Gesellschaft an sich und Familien, die es sich leicht machen können, wenn allgemeine Themen medizinalisiert werden könnten von der Überbetonung von ADHS als medizinisches Problem fälschlich profitieren.

Fazit: Das Buch von Amrei Wittwer ist zu Recht provokant und fordert häusliche und schulische Lebensumstände in den Blickpunkt zu stellen, wenn es um die kindliche Entwicklung geht. Die Autorin sollte sogar noch einen Schritt weiter gehen und soziodemografische, kulturelle und eben Gerechtigkeits- und Gleichheitsaspekte in Gesellschaften zum Gegenstand weiterer Betrachtungen machen. Insgesamt ist das Buch lesenswert, nicht immer muss man die Meinung der Autorin teilen aber es ist erfrischend die Überbetonung der Pharmakotherapie und der ach so omnipräsenten Genetik einmal hinterfragt zu sehen.

Wieland Kiess, Leipzig 